\title{
Rate calculation in two-dimensional barriers with colored noise
}

\author{
F. REVUELTA ${ }^{1,2, *}$, THOMAS BARTSCH ${ }^{3}$, R. M. BENITO ${ }^{1}$ and F. BORONDO ${ }^{2,4}$ \\ ${ }^{1}$ Grupo de Sistemas Complejos, Escuela Técnica Superior de Ingeniería Agronómica, Alimentaria y de Biosistemas, \\ Universidad Politécnica de Madrid, Avda. Puerta de Hierrro, 2-4, 28040 Madrid, Spain \\ ${ }^{2}$ Instituto de Ciencias Matemáticas (ICMAT), Cantoblanco, 28049 Madrid, Spain \\ ${ }^{3}$ Centre for Nonlinear Mathematics and Applications, Department of Mathematical Sciences, Loughborough \\ University, Loughborough LE11 3TU, United Kingdom \\ ${ }^{4}$ Departamento de Química, Universidad Autónoma de Madrid, Cantoblanco, 28049 Madrid, Spain \\ *Corresponding author. E-mail: fabio.revuelta@upm.es
}

\begin{abstract}
The identification of an optimal dividing surface that is free of recrossings is the most important requirement for transition state theory to be exact. This task is particularly difficult in the presence of non-Markovian friction, i.e., colored noise forces. In this paper, we report a novel geometric method that circumvents the recrossing problem and is able to (i) identify reactive trajectories exactly, and (ii) compute reaction rates in a system with two degrees of freedom driven by non-Markovian friction. The extension of our method to higher dimensional systems is also discussed.
\end{abstract}

Keywords. Rate theory; generalized Langevin equation; colored noise; correlations; non-Markovian friction.

PACS Nos 82.20.Db; 05.40.Ca; 05.45.2a; 34.10.+x

\section{Introduction}

Transition State Theory (TST) plays a central role in rate theory [1-3]. It provides a simple answer to two of the most fundamental questions in chemistry: it predicts if a trajectory will be reactive or not, and what the system reaction rate is.

The rate-limiting step in many reactions is the crossing of an energy barrier between reactant and product regions in phase space. Close to the saddle point at the top of the barrier an intermediate state, the transition state or activated complex, is formed. TST has been applied in many fields outside chemistry, such as celestial mechanics, and atomic and solid state physics.

The fundamental problem that TST faces is the identification of a dividing surface (DS) that separates reactants from products and is crossed only once by all reactive trajectories. For isolated systems, such a recrossing-free DS is provided by the Normally Hyperbolic Invariant Manifold (NHIM) [4-8]. However, the NHIM does not provide accurate results in condensed phase reactions, where the interaction with the environment cannot be neglected, and alternative procedures must be used. Variational TST is one of them [9]. It computes a DS by minimizing the number of recrossings.

In recent years, a strictly recrossing-free DS has been identified [10-14]. This DS moves randomly in the vicinity of the saddle point, attached to the socalled transition state (TS) trajectory. The TS trajectory, which was originally defined for parabolic barriers with white noise [10], has been recently extended to colored noise [15,16], laser-driven systems [17] and anharmonic barriers [18-21].

The study of realistic systems usually requires timeconsuming simulations because of the large number of surrounding particles that form the bath. The generalized Langevin equation (GLE) provides a simplified framework to adequately account for the interaction with the enviroment. This interaction is substituted by a time-correlated (colored) noise force, to account for the bath correlations, and a non-Markovian friction term. In the case of a harmonic barrier, the well-known Grote-Hynes theory (GHT) [22] provides the reaction rate. In the presence of anharmonicities, GHT no longer holds, and new approaches must be found. In this paper, we propose to use the stable manifold that separates reactive from non-reactive trajectories. We demonstrate that this manifold is easily defined in phase space and 
works well even in multi-dimensional systems. Our theory also accounts for the anharmonicities using a perturbative scheme and it has been successfully applied to compute the reaction rates of $\mathrm{LiNC} / \mathrm{LiCN}$ isomerizing system [15].

\section{The fundamental rate formula}

In this section, we summarize the fundamentals of reaction rate theory that will be used in the rest of the paper. For a more detailed discussion, see for example [23-25].

As mentioned above, TST is based on the assumption that there is a recrossing-free DS between reactants and products that is crossed once and only once by every reactive trajectory. In a two-dimensional reactive system with configuration space coordinates $x_{1}$ and $x_{2}$, the DS can be chosen by specifying the value $x_{1}=x_{1}^{\ddagger}$ of the reaction coordinate $x_{1}$. Reactant and product regions are defined by $x_{1}<x_{1}^{\ddagger}$ and $x_{1}>x_{1}^{\ddagger}$ respectively. TST is based on the flux-over-population expression for the reaction rate

$k=\frac{J}{N}$,

where $N$ is the average population of the reactant region and $J$ is the reactive flux across the DS. The DS in phase space can be parameterized by the velocity $v_{1}$ in the reactive mode, and the bath mode coordinates $x_{2}$ and $v_{2}$. (We ignore, for now, the auxiliary coordinates that are required to model correlated noise.) The reactive flux is then given by

$J=\left\langle v_{1} \chi_{\alpha}\left(v_{1}, x_{2}, v_{2}\right)\right\rangle_{\alpha, \mathrm{IC}}$,

where the average extends over all realizations $\alpha$ of the noise and over a stationary-state ensemble of initial conditions (IC) on the DS. The characteristic function $\chi_{\alpha}$ takes a value equal to 1 if the trajectory given by the IC $\left(x_{1}^{\ddagger}, v_{1}, x_{2}, v_{2}\right)$ is reactive if driven by the noise sequence $\alpha$ and 0 otherwise. It ensures that the trajectories included in the flux computation actually contribute to the rate. Its accurate computation poses the main dynamical challenge to a rate calculation, and therefore the principal building block of the theory developed here is the expression (eq. 35) for the characteristic function.

If the DS is recrossing-free, as postulated by TST, the reactive trajectories are precisely those that cross the DS from the reactant to the product side, i.e., with positive velocity
$\chi^{\mathrm{TST}}\left(v_{1}\right)= \begin{cases}1: & v_{1}>0, \\ 0: & v_{1}<0 .\end{cases}$

This assumption yields a reactive flux $J^{\mathrm{TST}}$ and a rate constant

$k^{\mathrm{TST}}=\frac{J^{\mathrm{TST}}}{N}$,

that always overestimates the true rate unless the DS is strictly recrossing-free. The extent to which a given system violates the no-recrossing assumption is measured by the transmission factor

$\kappa=\frac{k}{k^{\mathrm{TST}}} \leq 1$

The dynamical theory of the characteristic function that is to be developed below does not require any assumptions about the distribution of initial conditions; it applies equally to equilibrium and non-equilibrium systems. In practice, however, unless the friction caused by the heat bath is very weak, the stationary-state distribution of IC near the barrier is given by a Boltzmann equilibrium distribution. Deviations from this assumption give rise to the depopulation factor that is the crucial ingredient of the Mel'nikov-Meshkov [26] and PGH [27] turnover theories. A nontrivial depopulation factor can be combined with the present results if necessary, but for simplicity we will here assume an equilibrium distribution. The average over IC is then performed over an ensemble with probability density

$p\left(x_{1}, v_{1}, x_{2}, v_{2}\right)=\delta\left(x_{1}-x_{1}^{\ddagger}\right) \exp \left(-\frac{m v_{1}^{2}}{2 k_{\mathrm{B}} T}\right) p_{\perp}\left(x_{2}, v_{2}\right)$,

where $m$ is the particle mass and $p_{\perp}$ is a Boltzmann distribution

$p_{\perp}\left(x_{2}, v_{2}\right)=\frac{1}{Z} \exp \left(-\frac{m v_{2}^{2} / 2+U\left(x_{1}^{\ddagger}, x_{2}\right)}{k_{\mathrm{B}} T}\right)$

for the transverse coordinates and velocities, with the potential of mean force $U$. The factor $Z$ in eq. (7) is the partition function of the bath mode that ensures

$\int d x_{2} d v_{2} p_{\perp}\left(x_{1}^{\ddagger}, x_{2}, v_{2}\right)=1$.

Under the assumption of an equilibrium distribution, the TST flux can be evaluated analytically to give 
$J^{\mathrm{TST}}=\sqrt{\frac{k_{\mathrm{B}} T}{2 \pi m}}$.

The accurate evaluation of the true flux $J$ will be the main subject of this paper. To provide a benchmark for the perturbative calculations, we have computed the exact transmission factor numerically. To this end, initial conditions were randomly sampled from the equilibrium distribution (eq. 6), classical trajectories were numerically propagated using the algorithm described in Refs $[28,29]$ until their energy was far enough below the saddle point $\left(-7 k_{\mathrm{B}} T\right.$ in our case) so that they can be considered thermalized, and the necessary averages computed from the results.

\section{Model: The generalized Langevin equation}

The system that we have chosen to study is formed by a particle of mass $m$ that interacts with its surroundings and moves under the action of the potential

$U\left(x_{1}, x_{2}\right)=-\frac{1}{2} m \omega_{1}^{2} x_{1}^{2}+\frac{1}{2} m \omega_{2}^{2} x_{2}^{2}+m c x_{1}^{2} x_{2}^{2}$

shown in figure 1 . This potential has been studied by some of us in the presence of white noise $[12,13,21]$. The particle is said to react when it moves from one side of the barrier to the other, and $x_{1}$ changes its sign.

The GLE provides a simplified approach to describe the dynamics of a system that interacts with an isotropic external heat bath wich presents some correlations as

$m \ddot{\boldsymbol{x}}=-\nabla_{\boldsymbol{x}} U(\boldsymbol{x})-m \int_{-\infty}^{t} \gamma\left(t-t^{\prime}\right) \dot{\boldsymbol{x}}\left(t^{\prime}\right) d t^{\prime}+m \boldsymbol{R}_{\alpha}(t)$,

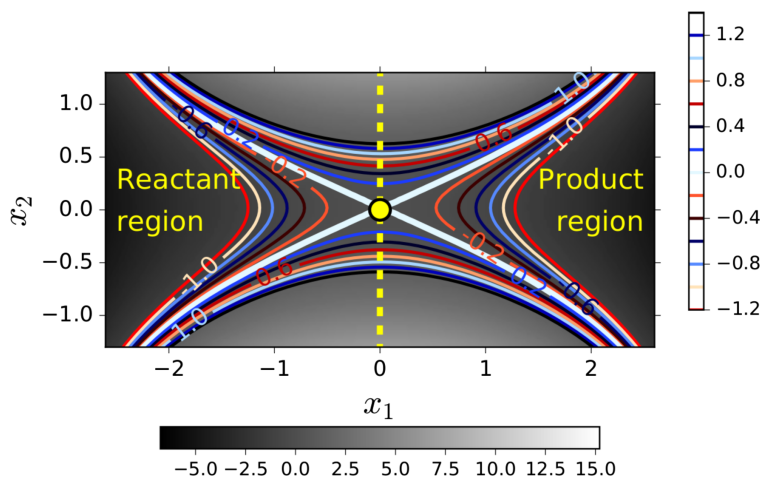

Figure 1. Potential energy surface given by eq. (10) for $m$ $=1, \omega_{1}=1, \omega_{2}=3$ and $c=-1$. The dividing surface (dashed yellow line), defined by $x_{1}^{\ddagger}=0$, crosses the saddle point (yellow dot) that sits at the barrier top $(0,0)$, and partitions the system into a reactant (left) and a product (right) region. where $\boldsymbol{x}=\left(x_{1}, x_{2}\right)^{\mathrm{T}}$ is a coordinate vector and $-\nabla_{\boldsymbol{x}} U(\boldsymbol{x})$ is the force produced by the potential (eq. 10). It can be split into harmonic and anharmonic terms as

$-\frac{\partial U\left(x_{1}, x_{2}\right)}{\partial x_{n}}=(-1)^{n-1} m \omega_{n}^{2} x_{n}+m f_{n}\left(x_{1}, x_{2}\right)$

with $n=1,2$. The components of the Gaussian noise force $\boldsymbol{R}_{\alpha}(t)$ have zero mean and are related to the friction kernel $\gamma(t)$ by the fluctuation-dissipation theorem

$\left\langle R_{\alpha, n}(0) R_{\alpha, n^{\prime}}(t)\right\rangle_{\alpha}=\frac{k_{\mathrm{B}} T \gamma(t)}{m} \delta_{n, n^{\prime}}$,

where $\langle\ldots\rangle_{\alpha}$ denotes an average over the different realizations $\alpha$ of the noise.

\subsection{The extended phase space}

The friction kernel of many realistic chemical reactions [30] has an exponential decay

$\gamma(t)=\frac{\gamma_{0}}{\tau} e^{-t / \tau}$,

with a characteristic correlation time $\tau$ and a damping strength $\gamma_{0}$. Then, as for a variety of other friction kernels with multi-exponential behaviour, the GLE (eq. 11) can be simplified to a system of differential equations on a finite dimensional extended phase space [15, 16,31-34] with two auxiliary coordinates

$\zeta_{1}(t)=-\int_{-\infty}^{t} \gamma\left(t-t^{\prime}\right) \dot{x}_{1}\left(t^{\prime}\right) d t^{\prime}$,

$\zeta_{2}(t)=-\int_{-\infty}^{t} \gamma\left(t-t^{\prime}\right) \dot{x}_{2}\left(t^{\prime}\right) d t^{\prime}$.

The lower limit $-\infty$ in the integrals (eq. 15) implies that the system was prepared in the remote past. It is crucial in order to make all of the six-dimensional phase space $\left(x_{1}, v_{1}, \zeta_{1}, x_{2}, v_{2}, \zeta_{2}\right)$ dynamically accessible [35]. In thermal equlibrium, $\zeta_{1}$ and $\zeta_{2}$ follow a Gaussian distribution with zero mean and correlations equal to

$\left\langle\zeta_{n}^{2}\right\rangle=\frac{k_{\mathrm{B}} T \gamma_{0}}{m \tau}, \quad\left\langle\zeta_{1} \zeta_{2}\right\rangle=0$

which are not correlated with the position $\mathbf{x}$, nor with the velocity $\mathbf{v}$ [33]. In this extended phase space, the GLE can be replaced by a new set of Equations of Motion (EoM) driven by a white noise source $\xi_{\alpha}=\left(\xi_{\alpha, 1}, \xi_{\alpha, 2}\right)^{T}$ :

$\dot{x}_{n}=v_{n}$,

$\dot{v}_{n}=-\frac{1}{m} \frac{\partial U\left(x_{1}, x_{2}\right)}{\partial x_{n}}+\zeta_{n}$ 
$\dot{\zeta}_{n}=-\frac{\gamma_{0}}{\tau} v_{n}-\frac{1}{\tau} \zeta_{n}+\xi_{\alpha, n}(t)$

with $n=1$ for the reaction coordinate and $n=2$ for the transverse dof. The components of the white noise source, $\boldsymbol{\xi}_{\alpha}(t)$, fulfill the fluctuation-dissipation theorem

$\left\langle\xi_{\alpha, n}(t) \xi_{\alpha, n^{\prime}}(s)\right\rangle_{\alpha}=\frac{2 k_{\mathrm{B}} T \gamma_{0}}{m \tau^{2}} \delta_{n n^{\prime}} \delta(t-s)$.

\subsection{Transition state trajectory and relative coordinates}

The EoM (eq. 17) of a system with a harmonic barrier $(f=0)$ and without noise $\left(\xi_{\alpha}(t)=0\right)$ can be rewritten as

$\dot{\boldsymbol{u}}_{n}=\mathbf{M}_{n} \boldsymbol{u}_{n}$,

where

$\mathbf{M}_{n}=\left(\begin{array}{ccc}0 & 1 & 0 \\ (-1)^{n-1} \omega_{n}^{2} & 0 & 1 \\ 0 & -\frac{\gamma_{0}}{\tau} & -\frac{1}{\tau}\end{array}\right), \quad$ and $\boldsymbol{u}_{n}=\left(\begin{array}{c}x_{n} \\ v_{n} \\ \zeta_{n}\end{array}\right)$

Each of the two coefficient matrices $\mathbf{M}_{1}$ and $\mathbf{M}_{2}$ has in general three different eigenvalues, at least one of them, $\lambda_{n, 0}$, real and the other two, $\lambda_{n, 1}$ and $\lambda_{n, 2}$, negative real or complex conjugate with negative real parts. The eigenvalues are the zeros of the characteristic polynomials of the matrices $\mathbf{M}_{n}$ and obey the Vieta relations $[15,16]$

$\lambda_{n, 0}+\lambda_{n, 1}+\lambda_{n, 2}=-\frac{1}{\tau}$,

$\lambda_{n, 0} \lambda_{n, 1}+\lambda_{n, 0} \lambda_{n, 2}+\lambda_{n, 1} \lambda_{n, 2}=\frac{\gamma_{0}}{\tau}+(-1)^{n} \omega_{n}^{2}$,

$\lambda_{n, 0} \lambda_{n, 1} \lambda_{n, 2}=(-1)^{n-1} \frac{\omega_{n}^{2}}{\tau}$.

The corresponding eigenvectors are $\tilde{\boldsymbol{u}}_{n, j}=\left(1, \lambda_{n, j}, \lambda_{n, j}^{2}\right.$ $\left.+(-1)^{n} \omega_{n}^{2}\right)^{T}$.

The eigenvalue $\lambda_{1,0}$ associated with the reactive dof has an eigenvector that describes an unstable direction in phase space as $0<\lambda_{1,0}<\omega_{1}^{2}$. The remaining eigenvalues are negative or have negative real parts and are therefore associated with eigenvectors that correspond to stable directions in phase space. Since $\lambda_{2,0}$ is negative real, a trajectory approaches the origin monotonically in the direction of $\tilde{\boldsymbol{u}}_{2,0}$ as $t \rightarrow \infty$. In the remaining stable directions, this approach to the origin can similarly take place monotonically, or in a non-monotonic manner, depending on whether the eigenvalues are real or complex.

In order to solve eq. (19), we now introduce the diagonal coordinates, $z_{n, i}$, by decomposing the phase space vectors

$\boldsymbol{u}_{n}=z_{n, 0} \tilde{\boldsymbol{u}}_{n, 0}+z_{n, 1} \tilde{\boldsymbol{u}}_{n, 1}+z_{n, 2} \tilde{\boldsymbol{u}}_{n, 2}$

in the basis of eigenvectors. The components of the transformation (22) read

$$
\begin{aligned}
x_{n}= & z_{n, 0}+z_{n, 1}+z_{n, 2}, \\
v_{n}= & \lambda_{n, 0} z_{n, 0}+\lambda_{n, 1} z_{n, 1}+\lambda_{n, 2} z_{n, 2} \\
\zeta_{n}= & {\left[\lambda_{n, 0}^{2}+(-1)^{n} \omega_{n}^{2}\right] z_{n, 0}+\left[\lambda_{n, 1}^{2}+(-1)^{n} \omega_{n}^{2}\right] z_{n, 1} } \\
& +\left[\lambda_{n, 2}^{2}+(-1)^{n} \omega_{n}^{2}\right] z_{n, 2}
\end{aligned}
$$

and its inverse is

$$
\begin{aligned}
& \left(\lambda_{n, j}-\lambda_{n, i}\right)\left(\lambda_{n, j}-\lambda_{n, k}\right) z_{n, j} \\
& \quad=\left[\lambda_{n, i} \lambda_{n, k}+(-1)^{n-1} \omega_{n}^{2}\right] x_{n}-\left(\lambda_{n, i}+\lambda_{n, k}\right) v_{n}+\zeta_{n},
\end{aligned}
$$

where $i, j, k=0,1,2$ are distinct. The EoM (eq. 17) in the diagonal coordinates are

$\dot{z}_{n, j}=\lambda_{n, j} z_{n, j}+K_{n, j} f_{n}\left(x_{1}, x_{2}\right)+\frac{1}{F_{n, j}} \xi_{\alpha, n}(t)$,

with the abbreviations

$F_{n, j}=\left(\lambda_{n, j}-\lambda_{n, i}\right)\left(\lambda_{n, j}-\lambda_{n, k}\right), K_{n, j}=-\frac{\lambda_{n, i}+\lambda_{n, k}}{F_{n, j}}$.

In order to solve eq. (25), we make a time-dependent shift to the relative coordinates

$\Delta z_{n, j}(t)=z_{n, j}(t)-z_{n, j}^{\ddagger}(t)$,

where $z_{n, j}^{\ddagger}$ are the six components of the TS trajectory,

$z_{n, i}^{\ddagger}(t)=\frac{1}{F_{n, i}} S\left[\lambda_{n, i}, \xi_{n, \alpha} ; t\right]$,

with $S[\mu, g ; t] \equiv S_{t^{\prime}}[\mu, g ; t]$ defined as

$S_{t^{\prime}}[\mu, g ; t]= \begin{cases}-\int_{t}^{\infty} g\left(t^{\prime}\right) e^{\mu\left(t-t^{\prime}\right)} d t^{\prime}: & \operatorname{Re} \mu>0, \\ +\int_{-\infty}^{t} g\left(t^{\prime}\right) e^{\mu\left(t-t^{\prime}\right)} d t^{\prime} & : \operatorname{Re} \mu<0 .\end{cases}$ 
The functions $z_{n, i}^{*}(t)$ solve the EoM (eq. 25) in the harmonic limit, $f=0$. They are random variables with zero mean whose correlation functions can be deduced from eq. (28):

$$
\begin{aligned}
\left\langle z_{1,0}^{\ddagger}(t) z_{1,0}^{\ddagger}(0)\right\rangle_{\alpha} & =\frac{k_{\mathrm{B}} T \gamma_{0}}{m \tau^{2} \lambda_{1,0} F_{1,0}^{2}} e^{-\lambda_{1,0} t}, \\
\left\langle z_{1,0}^{\ddagger}(t) z_{1, i}^{\ddagger}(0)\right\rangle_{\alpha} & =\left\langle z_{1, k}^{\ddagger}(t) z_{2, l}^{\ddagger}(0)\right\rangle_{\alpha}=0, \\
\left\langle z_{1, i}^{\ddagger}(t) z_{1,0}^{\ddagger}(0)\right\rangle_{\alpha} & =\frac{2 k_{\mathrm{B}} T \gamma_{0}\left(e^{-\lambda_{1,0} t}-e^{\lambda_{1, i} t}\right)}{m \tau^{2}\left(\lambda_{1,0}+\lambda_{1, i}\right) F_{1,0} F_{1, i}}, \\
\left\langle z_{1, i}^{\ddagger}(t) z_{1, j}^{\ddagger}(0)\right\rangle_{\alpha} & =-\frac{2 k_{\mathrm{B}} T \gamma_{0}}{m \tau^{2}\left(\lambda_{1, i}+\lambda_{1, j}\right) F_{1, i} F_{1, j}} e^{\lambda_{1, i} t}, \\
\left\langle z_{2, k}^{\ddagger}(t) z_{2, l}^{\ddagger}(0)\right\rangle_{\alpha} & =-\frac{2 k_{\mathrm{B}} T \gamma_{0}}{m \tau^{2}\left(\lambda_{2, j}+\lambda_{2, k}\right) F_{2, j} F_{2, k}} e^{\lambda_{2, j} t},
\end{aligned}
$$

where $i, j=1,2, k, l=0,1,2$, and $t \geq 0$.

In relative coordinates (eq. 27), the EoM (eq. 25) reduce to

$\Delta \dot{z}_{n, j}=\lambda_{n, j} \Delta z_{n, j}+K_{n, j} f_{n}\left(x_{1}, x_{2}\right)$,

where

$x_{1}=x_{1}^{\ddagger}+\Delta z_{1,0}+\Delta z_{1,1}+\Delta z_{1,2}$,
$x_{2}=x_{2}^{\ddagger}+\Delta z_{2,0}+\Delta z_{2,1}+\Delta z_{2,2}$.

Eq. (31) are coupled only through the force anharmonicities $f_{n}$, which makes them amenable to a perturbative treatment in the case of weak anharmonicity.

The solution of the EoM (eq. 31) is given by

$$
\begin{aligned}
\Delta z_{1,0}(t) & =C_{0} e^{\lambda_{1,0} t}+K_{1,0} S\left[\lambda_{1,0}, f_{1}\left(x_{1}, x_{2}\right) ; t\right], \\
\Delta z_{n, j}(t) & =\Delta z_{n, j}(0) e^{\lambda_{n, j} t}+K_{n, j} \bar{S}\left[\lambda_{n, j}, f_{n}\left(x_{1}, x_{2}\right) ; t\right],
\end{aligned}
$$

where $n=1,2, j=0,1,2$ and $(n, j) \neq(1,0)$. The functional $\bar{S}$ is defined as

$\bar{S}_{t^{\prime}}[\mu, g ; t]=\int_{0}^{t} g\left(t^{\prime}\right) e^{\mu\left(t-t^{\prime}\right)} d t^{\prime}$.

It satisfies the differential equation

$\frac{d}{d t} \bar{S}[\mu, g ; t]=\mu \bar{S}[\mu, g ; t]+g(t)$

and the IC $\bar{S}[\mu, g ; 0]=0$. All trajectories with $C_{0} \neq 0$ will fall in the reactant or product well after suficiently long time, depending on the sign of $C_{0}$. The critical situation where $C_{0}=0$ defines the stable manifold which encodes all the necessary information to determine whether a given trajectory is reactive or not, as described in the section below.

\section{The geometric separatrix for reactivity}

By setting $C_{0}=0$ in eq. (33a), we obtain a trajectory that lies on the stable manifold. This object is fivedimensional, like the DS defined by $x_{1}=0$. The intersection of the previous structures in a six-dimensional phase space is a four-dimensional surface that partitions the DS into reactive and nonreactive regions. It can be described as the critical velocity function $V^{\ddagger}=V^{\ddagger}\left(\zeta_{1}, x_{2}, v_{2}, \zeta_{2}\right)$, which can be used to circunvent the problem on the existence of a recrossing-free DS as all reactive trajectories have initial velocities larger than the critical one, $V^{\ddagger}$. The characteristic function of reactive trajectories can therefore be expressed exactly as

$\chi_{\alpha}\left(v_{1}, \zeta_{1}, x_{2}, v_{2}, \zeta_{2}\right)= \begin{cases}1 & : v_{1}>V^{\ddagger}\left(\zeta_{1}, x_{2}, v_{2}, \zeta_{2}\right), \\ 0 & : v_{1}<V^{\ddagger}\left(\zeta_{1}, x_{2}, v_{2}, \zeta_{2}\right) .\end{cases}$

Since the critical velocity, or the stable manifold that gives rise to it, identifies reactive trajectories accurately, it can be used to circunvent the problematic search of a recrossing-free DS.

\subsection{The critical velocity}

To find the critical velocity of the trajectories lying at the DS, we have to solve eq. (33) with the IC $x_{1}(0)=0$, $\zeta_{1}(0), x_{2}(0), v_{2}(0)$ and $\zeta_{2}(0)$. As in Refs $[20,21]$, the IC $z_{1,1}(0)$ and $z_{1,2}(0)$ of the stable coordinates in the reactive dof must be adapted in each step of perturbation theory in order to fulfill the conditions

$$
\begin{aligned}
x_{1}(0)= & 0=z_{1,0}(0)+z_{1,1}(0)+z_{1,2}(0) \\
\zeta_{1}(0)= & \left(\lambda_{1,0}^{2}-\omega_{1}^{2}\right) z_{1,0}(0)+\left(\lambda_{1,1}^{2}-\omega_{1}^{2}\right) z_{1,1}(0) \\
& +\left(\lambda_{1,2}^{2}-\omega_{1}^{2}\right) z_{1,2}(0)
\end{aligned}
$$

which are satisfied by

$z_{1, j}(0)=\frac{\zeta_{1}(0)+\left(\lambda_{1, k}^{2}-\lambda_{1,0}^{2}\right) z_{1,0}(0)}{\lambda_{1, j}^{2}-\lambda_{1, k}^{2}}$,

with $k=1,2$ for $j=2,1$. The unstable coordinate $z_{1,0}(0)$ and the critical velocity

$$
\begin{aligned}
V^{\ddagger}=v_{1}(0) & =\lambda_{1,0} z_{1,0}(0)+\lambda_{1,1} z_{1,1}(0)+\lambda_{1,2} z_{1,2}(0) \\
& =\frac{1}{K_{1,0}} z_{1,0}(0)+\frac{1}{\lambda_{1,1}+\lambda_{1,2}} \zeta_{1}(0)
\end{aligned}
$$


are determined such that the trajectory lies on the stable manifold. The IC for the transversal dof are fixed by imposing the conditions

$$
\begin{aligned}
x_{2}(0)= & z_{2,0}(0)+z_{2,1}(0)+z_{2,2}(0) \\
v_{2}(0)= & \lambda_{2,0} z_{2,0}(0)+\lambda_{2,1} z_{2,1}(0)+\lambda_{2,2} z_{2,2}(0), \\
\zeta_{2}(0)= & \left(\lambda_{2,0}^{2}+\omega_{2}^{2}\right) z_{2,0}(0)+\left(\lambda_{2,1}^{2}+\omega_{2}^{2}\right) z_{2,1}(0) \\
& +\left(\lambda_{2,2}^{2}+\omega_{2}^{2}\right) z_{2,2}(0)
\end{aligned}
$$

It follows that

$z_{2, i}(0)=\frac{\lambda_{2, j} \lambda_{2, k}-\omega_{2}^{2}}{F_{2, i}} x_{2}(0)+K_{2, i} \nu_{2}(0)+\frac{\zeta_{2}(0)}{F_{2, i}}$,

where $i \neq j \neq k$.

For anharmonic barriers, we expand the TS trajectory in powers of the coupling constant $c$ around its harmonic approximation:

$$
\begin{aligned}
X_{1}(t)= & x_{1}^{\ddagger}(t)+\Delta z_{1,1}(0) e^{\lambda_{1,1} t}+\Delta z_{1,2}(0) e^{\lambda_{1,2} t}, \\
X_{2}(t)= & x_{2}^{\ddagger}(t)+\Delta z_{2,0}(0) e^{\lambda_{2,0} t}+\Delta z_{2,1}(0) e^{\lambda_{2,1} t} \\
& +\Delta z_{2,2}(0) e^{\lambda_{2,2} t} .
\end{aligned}
$$

The coordinates

$x_{1}(t)=X_{1}(t)+c \Delta x_{1}^{(1)}(t)+c^{2} \Delta x_{1}^{(2)}(t)+\ldots$,
$x_{2}(t)=X_{2}(t)+c \Delta x_{2}^{(1)}(t)+c^{2} \Delta x_{2}^{(2)}(t)+\ldots$

and the critical velocity (eq. 38)

$$
V^{\ddagger}=V^{\ddagger(0)}+c V^{\ddagger(1)}+c^{2} V^{\ddagger(2)}+\ldots
$$

can similarly be expanded.

Substituting the perturbations (eq. 42) into the anharmonic terms of the force appearing in eq. (12), we get

$f_{1}=-2 c x_{1} x_{2}^{2}=c f_{1}^{(1)}+c^{2} f_{1}^{(2)}+\ldots$,

$f_{2}=-2 c x_{1}^{2} x_{2}=c f_{2}^{(1)}+c^{2} f_{2}^{(2)}+\ldots$,

where the perturbation terms of order 1 and 2 are given by

$f_{j}^{(1)}=-2 X_{i} X_{j}^{2}$

$f_{j}^{(2)}=-2\left(X_{i}^{2} \Delta x_{j}^{(j)}+2 X_{1} X_{2} \Delta x_{i}^{(1)}\right)$,

with $i, j=1,2, i \neq j$, and

$\Delta x_{n}^{(k)}=\Delta z_{n, 0}^{(k)}+\Delta z_{n, 1}^{(k)}+\Delta z_{n, 2}^{(k)}$.
Using eq. (33), it can easily be demonstrated that

$\Delta z_{1,0}^{(k)}(t)=K_{1,0} S\left[\lambda_{1,0}, f_{1}^{(k)} ; t\right]$,

$\Delta z_{n, j}^{(k)}(t)=\Delta z_{n, j}^{(k)}(0) e^{\lambda_{n, j} t}+K_{n, j} \bar{S}\left[\lambda_{n, j}, f_{n}^{(k)} ; t\right]$

for $(n, j) \neq(1,0)$. Therefore, the computation of $\Delta x_{n}^{(k)}$ requires the previous computation of only the lower order perturbative corrections.

From eqs (37-38) one obtains

$V^{\ddagger(k)}=\frac{1}{K_{1,0}} \Delta z_{1,0}^{(k)}(0)$

and then $\Delta z_{1, j}^{(k)}(0)=K_{1, j} V^{\ddagger(k)}$, for $j=1,2$. These equations allow one to determine the corrections to the critical velocity from eq. (48a), and then the corrections to the IC $\Delta z_{1, j}^{(k)}(0)$. Since the IC $x_{2}(0), v_{2}(0), \zeta_{2}(0)$ in the transverse dof are all fixed as arguments to the function $V^{\ddagger}\left(\zeta_{1}, x_{2}, v_{2}, \zeta_{2}\right)$ that must be computed, it follows from eq. (40) that $\Delta z_{2, j}^{(k)}(0)=0$ for $j=0,1,2$ and $k \geq 1$.

We are now in a position to obtain explicit analytical expressions for the perturbative corrections to the critical velocity. The lowest order term is given by the harmonic approximation to eq. (38)

$V^{\ddagger(0)}=\frac{1}{K_{1,0}} z_{1,0}^{\ddagger}(0)+\frac{1}{\lambda_{1,1}+\lambda_{1,2}} \zeta_{1}(0)$,

while the first correction is obtained by setting $k=1$ in eq. (49) as

$V^{\ddagger(1)}=\frac{1}{K_{1,0}} \Delta z_{1,0}^{(1)}(0)=-2 S\left[\lambda_{1,0}, X_{1} X_{2}^{2} ; 0\right]$.

The computation of the second order perturbative term of the critical velocity is more cumbersome as the first order corrections to the stable coordinates given by

$$
\begin{aligned}
\Delta z_{1, i}^{(1)}(t)= & \Delta z_{1, i}^{(1)}(0) e^{\lambda_{1, i} t}+K_{1, i} \bar{S}\left[\lambda_{1, i}, f_{1}^{(1)} ; t\right] \\
= & -2 K_{1, i} S\left[\lambda_{1,0}, X_{1} X_{2}^{2} ; 0\right] e^{\lambda_{1, i} t} \\
& -2 K_{1, i} \bar{S}\left[\lambda_{1, i}, X_{1} X_{2}^{2} ; t\right] \\
\Delta z_{2, j}^{(1)}(t)= & K_{2, j} \bar{S}\left[\lambda_{2, j}, f_{2}^{(1)} ; t\right] \\
= & -2 K_{2, j} \bar{S}\left[\lambda_{2, j}, X_{1}^{2} X_{2} ; t\right]
\end{aligned}
$$

with $i=1,2$ and $j=0,1,2$, are also more complicated. The first order corrections to the original coordinates are given by 


$$
\begin{aligned}
\Delta x_{1}^{(1)}(t)=\Delta z_{1,0}^{(1)}(t)+\Delta z_{1,1}^{(1)}(t)+\Delta z_{1,2}^{(1)}(t) \\
=-2 K_{1,0} S\left[\lambda_{1,0}, X_{1} X_{2}^{2} ; t\right]-2 K_{1,1} S\left[\lambda_{1,0}, X_{1} X_{2}^{2} ; 0\right] e^{\lambda_{1,1} t} \\
\quad-2 K_{1,1} \bar{S}\left[\lambda_{1,1}, X_{1} X_{2}^{2} ; t\right]-2 K_{1,2} S\left[\lambda_{1,0}, X_{1} X_{2}^{2} ; 0\right] e^{\lambda_{1,2} t} \\
\quad-2 K_{1,2} \bar{S}\left[\lambda_{1,2}, X_{1} X_{2}^{2} ; t\right],
\end{aligned}
$$

and

$$
\begin{aligned}
& \Delta x_{2}^{(1)}(t)=\Delta z_{2,0}^{(1)}(t)+\Delta z_{2,1}^{(1)}(t)+\Delta z_{2,2}^{(1)}(t) \\
& =-2 K_{2,0} \bar{S}\left[\lambda_{2,0}, X_{1}^{2} X_{2} ; t\right]-2 K_{2,1} \bar{S}\left[\lambda_{2,1}, X_{1}^{2} X_{2} ; t\right] \\
& \quad-2 K_{2,2} \bar{S}\left[\lambda_{2,2}, X_{1}^{2} X_{2} ; t\right] .
\end{aligned}
$$

By combination of eqs (44a), (48a), (49), (53) and (54), one finally arrives at the second order correction to the critical velocity, which is given by

$$
\begin{aligned}
& V^{\ddagger(2)}=\frac{1}{K_{1,0}} \Delta z_{1,0}^{(2)}(0) \\
& =-2 S\left[\lambda_{1,0}, X_{2}^{2} \Delta x_{1}^{(1)}+2 X_{1} X_{2} \Delta x_{2}^{(1)} ; 0\right] \\
& =4 S_{t}\left[\lambda_{1,0}, X_{2}^{2}(t)\left(K_{1,0} S\left[\lambda_{1,0}, X_{1} X_{2}^{2} ; t\right]\right.\right. \\
& \left.+K_{1,1} S\left[\lambda_{1,0}, X_{1} X_{2}^{2} ; 0\right] e^{\lambda_{1,1} t}+K_{1,2} S\left[\lambda_{1,0}, X_{1} X_{2}^{2} ; 0\right] e^{\lambda_{1,2} t}\right) \\
& +K_{1,1} \bar{S}\left[\lambda_{1,1}, X_{1} X_{2}^{2} ; t\right]+K_{1,2} \bar{S}\left[\lambda_{1,2}, X_{1} X_{2}^{2} ; t\right] \\
& +2 X_{1}(t) X_{2}(t)\left(K_{2,0} \bar{S}\left[\lambda_{2,0}, X_{1}^{2} X_{2} ; t\right]\right. \\
& \left.\left.+K_{2,1} \bar{S}\left[\lambda_{2,1}, X_{1}^{2} X_{2} ; t\right]+K_{2,2} \bar{S}\left[\lambda_{2,2}, X_{1}^{2} X_{2} ; t\right]\right) ; 0\right]
\end{aligned}
$$

\section{The transmission factor}

As reported in Ref. [16] , the transmission factor for a system of 2 dof can be computed as

$\kappa=\left\langle\exp \left(-\frac{V^{\ddagger 2}}{2 k_{\mathrm{B}} T}\right)\right\rangle_{\alpha \zeta \perp}$,

where $\alpha$ represents an average over different noise ensembles, $\zeta$ an average carried out over the IC $\zeta_{1}(0)$ and $\zeta_{2}(0)$, and $\perp$ an average over the transversal coordinates $x_{2}(0)$ and $v_{2}(0)$.

Substituting the expansion of the critical velocity given by eq. (43) in eq. (56), we get an expansion of the transmission factor in the coupling constant $c$ as

$\kappa=\kappa^{(0)}+c \kappa^{(1)}+c^{2} \kappa^{(2)}+\ldots$.

The lowest order terms are given by

$$
\begin{aligned}
\kappa^{(0)} & =\langle P\rangle_{\alpha \zeta \perp}, \\
\kappa^{(1)} & =-\frac{m}{k_{\mathrm{B}} T}\left\langle P V^{\ddagger(0)} V^{\ddagger(1)}\right\rangle_{\alpha \zeta \perp},
\end{aligned}
$$

$$
\begin{aligned}
\kappa^{(2)}= & \frac{m^{2}}{2\left(k_{\mathrm{B}} T\right)^{2}}\left\langle P V^{\ddagger(0) 2} V^{\ddagger(1) 2}\right\rangle_{\alpha \zeta \perp} \\
& -\frac{m}{k_{\mathrm{B}} T}\left\langle P V^{\ddagger(0)} V^{\ddagger(2)}\right\rangle_{\alpha \zeta \perp}-\frac{m}{2 k_{\mathrm{B}} T}\left\langle P V^{\ddagger(1) 2}\right\rangle_{\alpha \zeta \perp},
\end{aligned}
$$

with $P=\exp \left(-\frac{m V^{\ddagger}(0) 2}{2 k_{\mathrm{B}} T}\right)$, and the perturbative terms of the critical velocity are taken from eqs (50), (51) and (55). The evaluation of the perturbative terms of the transmission factor (eq. 58) requires the evaluation of averages $\langle P(\ldots)\rangle_{\alpha \zeta \perp}$ as discussed in the next subsection.

\subsection{Distorted correlation functions}

The averages appearing in eq. (58) can be rewritten in the form

$\langle P(\ldots)\rangle_{\alpha \zeta}=\frac{\lambda_{1,0}}{\omega_{1}}\langle\ldots\rangle_{0}$,

where the random variables $w_{1}=V^{\ddagger(0)}, w_{2}, \ldots$ appearing in (...) follow a multi-dimensional Gaussian distribution with zero mean and covariance matrix $\Sigma$. The factor $P$ can be absorbed [20,21] into a modified covariance matrix $\Sigma_{0}$ that satisfies

$\Sigma_{0}^{-1}=\Sigma^{-1}+\frac{m}{k_{\mathrm{B}} T} J, \quad$ with $J=\left(\begin{array}{cccc}1 & 0 & 0 & \ldots \\ 0 & 0 & 0 & \ldots \\ 0 & 0 & 0 & \ldots \\ \vdots & \vdots & \vdots & \ddots\end{array}\right)$,

or

$$
\begin{aligned}
\Sigma_{0} & =\Sigma-\frac{m}{k_{\mathrm{B}} T+m \sigma^{2}} \Sigma J \Sigma \\
& =\Sigma-\frac{m}{k_{\mathrm{B}} T+m \sigma^{2}} \frac{\lambda_{1,0}^{2}}{\omega_{1}^{2}} \Sigma J \Sigma,
\end{aligned}
$$

where

$$
\begin{aligned}
\sigma^{2}= & \left\langle V^{\ddagger(0) 2}\right\rangle_{\alpha \zeta} \\
= & \frac{\left(\lambda_{1,0}-\lambda_{1,1}\right)^{2}\left(\lambda_{1,0}-\lambda_{1,2}\right)^{2}}{\left(\lambda_{1,1}+\lambda_{1,2}\right)^{2}}\left\langle z_{1,0}^{\ddagger 2}(0)\right\rangle_{\alpha} \\
& +\frac{1}{\left(\lambda_{1,1}+\lambda_{1,2}\right)^{2}}\left\langle\zeta_{1}^{2}(0)\right\rangle_{\zeta} \\
= & \frac{k_{\mathrm{B}} T \gamma_{0}}{m \lambda_{1,0}\left(1+\lambda_{1,0} \tau\right)} .
\end{aligned}
$$

In the derivation of eq. (62) from eq. (50) the following were taken into account:

- $z_{1,0}^{\ddagger}(0)$ depends solely on the noise and $\zeta(0)$ solely on the IC; in particular

$$
\left\langle z_{1,0}^{\ddagger}(0) \zeta(0)\right\rangle_{\alpha, \zeta}=\left\langle z_{1,0}^{\ddagger}(0)\right\rangle_{\alpha}\langle\zeta(0)\rangle_{\zeta}=0 .
$$


- According to the Vieta relation (eq. 21),

$$
\left(\lambda_{1,1}+\lambda_{1,2}\right) \tau=-\left(1+\lambda_{1,0} \tau\right) .
$$

- Since $P_{1}\left(\lambda_{1,0}\right)=0$,

$$
\begin{aligned}
& \frac{\lambda_{1,0}^{2}}{\omega_{1}^{2}}\left[\lambda_{1,0}\left(1+\lambda_{1,0} \tau\right)+\gamma_{0}\right] \\
& \quad=-\frac{\tau}{\omega_{1}^{2}} \lambda_{1,0} P_{1}\left(\lambda_{1,0}\right)+\lambda_{1,0}\left(1+\lambda_{1,0} \tau\right) \\
& \quad=\lambda_{1,0}\left(1+\lambda_{1,0} \tau\right) .
\end{aligned}
$$

The average (eq. 59) of a product of two Gaussian random variables is then given by

$$
\left\langle w_{i} w_{j}\right\rangle_{0}=\left\langle w_{i} w_{j}\right\rangle_{\alpha \zeta}-\frac{m}{k_{\mathrm{B}} T} \frac{\lambda_{1,0}^{2}}{\omega_{1}^{2}}\left\langle V^{\ddagger(0)} w_{i}\right\rangle_{\alpha \zeta}\left\langle V^{\ddagger(0)} w_{j}\right\rangle_{\alpha \zeta},
$$

which allows one to obtain the moments of the distorted Gaussian distribution, once those of the original Gaussian are known.

By setting $w_{i}=V^{\ddagger(0)}$, and taking into account that $\lambda_{1,0}^{2}\left(1+\lambda_{1,0} \tau\right)=\left(\omega_{1}^{2} \tau-\gamma_{0}\right) \lambda_{1,0}+\omega_{1}^{2}$, we find

$$
\begin{aligned}
\left\langle V^{\ddagger(0)} w_{j}\right\rangle_{0} & =\left\langle V^{\ddagger(0)} w_{j}\right\rangle_{\alpha \zeta}\left(1-\frac{m}{k_{\mathrm{B}} T} \frac{\lambda_{1,0}^{2}}{\omega_{1}^{2}}\left\langle V^{\ddagger(0) 2}\right\rangle_{\alpha \zeta}\right) \\
& =\left\langle V^{\ddagger(0)} w_{j}\right\rangle_{\alpha \zeta} \frac{\omega_{1}^{2}\left(1+\lambda_{1,0} \tau\right)-\lambda_{1,0} \gamma_{0}}{\omega_{1}^{2}\left(1+\lambda_{1,0} \tau\right)} \\
& =\frac{\lambda_{1,0}^{2}}{\omega_{1}^{2}}\left\langle V^{\ddagger(0)} w_{j}\right\rangle_{\alpha \zeta} .
\end{aligned}
$$

If $w_{i}=w_{j}=V^{\ddagger(0)}$, eq. (65) reduces to

$$
\left\langle V^{\ddagger(0) 2}\right\rangle_{0}=\frac{\lambda_{1,0}^{2}}{\omega_{1}^{2}}\left\langle V^{\ddagger(0) 2}\right\rangle_{\alpha \zeta}=\frac{k_{\mathrm{B}} T}{m}\left(1-\frac{\lambda_{1,0}^{2}}{\omega_{1}^{2}}\right),
$$

as $\gamma_{0}=-\lambda_{1,0}^{2} \tau-\lambda_{1,0}+\omega_{1}^{2} \tau+\frac{\omega_{1}^{2}}{\lambda_{1,0}}=\left(\frac{1}{\lambda_{1,0}}+\tau\right)\left(\omega_{1}^{2}-\lambda_{1,0}^{2}\right)$.

Distorted averages involving more than two factors of $V^{\ddagger(0)}, X_{1}(t)$ and $X_{2}(t)$, like those in eq. (56), can be reduced to the correlation functions in eqs (66), (68a) and (68b) by Isserlis' theorem, e.g.

$$
\begin{aligned}
\left\langle w_{1} w_{2} w_{3} w_{4}\right\rangle_{0}= & \left\langle w_{1} w_{2}\right\rangle_{0}\left\langle w_{3} w_{4}\right\rangle_{0}+\left\langle w_{1} w_{3}\right\rangle_{0}\left\langle w_{2} w_{4}\right\rangle_{0} \\
& +\left\langle w_{1} w_{4}\right\rangle_{0}\left\langle w_{2} w_{3}\right\rangle_{0} .
\end{aligned}
$$

This expression contains a sum over all possible pairings of the four factors. Other even order moments can be evaluated in a similar way. All odd order moments are zero. However, as the anharmonic potential (eq. 10) is even, no odd order moments arise in the calculation. If the potential contained cubic terms, the leading order rate correction would only be obtained in second order perturbation theory.

For our purposes, the following modified correlation functions are needed:

$$
\begin{aligned}
& \left\langle V^{\ddagger(0)} X_{1}(t)\right\rangle_{0}=\frac{k_{\mathrm{B}} T}{m \lambda_{1,0}}\left[e^{-\lambda_{1,0} t}\right. \\
& \left.+\frac{\lambda_{1,2} \tau\left(\lambda_{1,0}+\lambda_{1,2}\right)}{\left(\lambda_{1,2}-\lambda_{1,1}\right)} e^{\lambda_{1,1} t}+\frac{\lambda_{1,1} \tau\left(\lambda_{1,0}+\lambda_{1,1}\right)}{\left(\lambda_{1,1}-\lambda_{1,2}\right)} e^{\lambda_{1,2} t}\right], \\
& \left\langle X_{1}(t) X_{1}(0)\right\rangle_{0}=\frac{k_{\mathrm{B}} T}{m}\left[\frac{K_{1,0}}{\lambda_{1,0}} e^{-\lambda_{1,0} t}+\frac{K_{1,1}}{\lambda_{1,1}} e^{\lambda_{1,1} t}\right. \\
& +\frac{K_{1,2}}{\lambda_{1,2}} e^{\lambda_{1,2} t}+\frac{\lambda_{1,0} \lambda_{1,2}+\omega_{1}^{2}}{F_{1,1} \omega_{1}^{2}}\left(e^{-\lambda_{1,0} t}+e^{\lambda_{1,1} t}\right) \\
& \left.+\frac{1}{\omega_{1}^{2}} e^{-(t+s) \lambda_{1,0}}+\frac{\lambda_{1,0} \lambda_{1,1}+\omega_{1}^{2}}{F_{1,2} \omega_{1}^{2}}\left(e^{-\lambda_{1,0} t}+e^{\lambda_{1,2} t}\right)\right], \\
& \left\langle X_{2}(t) X_{2}(0)\right\rangle_{0 \zeta \perp}=-\frac{k_{\mathrm{B}} T}{m} \frac{K_{2,0}}{\lambda_{2,0}} e^{\lambda_{2,0}|t s|} \\
& \quad-\frac{k_{\mathrm{B}} T}{m} \frac{K_{2,1}}{\lambda_{2,1}} e^{\lambda_{2,1}|t|}-\frac{k_{\mathrm{B}} T}{m} \frac{K_{2,2}}{\lambda_{2,1}} e^{\lambda_{2,1}|t|} .
\end{aligned}
$$

Eq. (68c) has been obtained by taking into account that the IC of the transversal direction follow the Gaussian distribution given by eq. (7), and therefore

$$
\begin{aligned}
\left\langle x_{2}^{2}(0)\right\rangle_{0 \zeta \perp} & =\frac{\left\langle v_{2}^{2}(0)\right\rangle_{0 \zeta \perp}}{m \omega_{2}^{2}}=\frac{k_{\mathrm{B}} T}{m \omega_{2}^{2}}, \quad\left\langle x_{2}(0) v_{2}(0)\right\rangle_{0 \zeta \perp} \\
& =0 .
\end{aligned}
$$

Finally,

$$
\left\langle V^{\ddagger(0)} X_{2}(t)\right\rangle_{0 \zeta \perp}=\left\langle X_{1}(t) X_{2}(s)\right\rangle_{0 \zeta \perp}=0
$$

since $X_{1}(t)$ and $X_{2}(t)$ depend on different and uncorrelated components of the Gaussian noise $\boldsymbol{\xi}_{\alpha}(t)$. In particular, the presence of the factor $P$ in eq. (58) has no influence on averages over the transverse dof. 


\subsection{Anharmonic corrections to the transmission factor}

The lowest order term of the transmission factor equals the well-known Grote-Hynes expression, which determines the rate over a one-dimensional harmonic barrier as

$\kappa^{(0)}=\langle P\rangle_{\alpha \zeta \perp}=\langle P\rangle_{\alpha}=\frac{\lambda_{1,0}}{\omega_{1}}$.

The first order correction to $\kappa^{(0)}$ can be calculated combining eqs. (58b) and (68) to yield

$\kappa^{(1)}=-\frac{k_{\mathrm{B}} T \kappa^{(0)}\left(1-\kappa^{(0) 2}\right)\left(\kappa^{(0) 2}+v^{2}\right)}{\left[\kappa^{(0) 4}+\kappa^{(0) 2}\left(v^{2}-1\right)+v^{2}\right] m \omega_{2}^{2} \omega_{1}^{2}}$, with $v^{2}=\lambda_{1,0}\left(1+\lambda_{1,0} \tau\right) /\left(\omega_{1}^{2} \tau\right)$. Eq. (71) agrees with the white noise limit

$\kappa^{(1)}(\nu \rightarrow \infty)=-\frac{k_{\mathrm{B}} T \gamma_{0} \kappa^{(0) 2}}{\left(1+\kappa^{(0) 2}\right) m \omega_{2}^{2} \omega_{1}^{3}}$,

obtained in Ref. [21], since $\gamma_{0}=\left(1-\kappa^{(0) 2}\right) \omega_{1} / \kappa^{(0)}$ in that limit. (The corresponding eq. (85) in Ref. [21] contains a misprint.)

An analytical expression for the second order correction (eq. 58c) can be similarly obtained, but we do not present it here explicitly as it is very complicated. The interested reader can find it in Ref. [16].

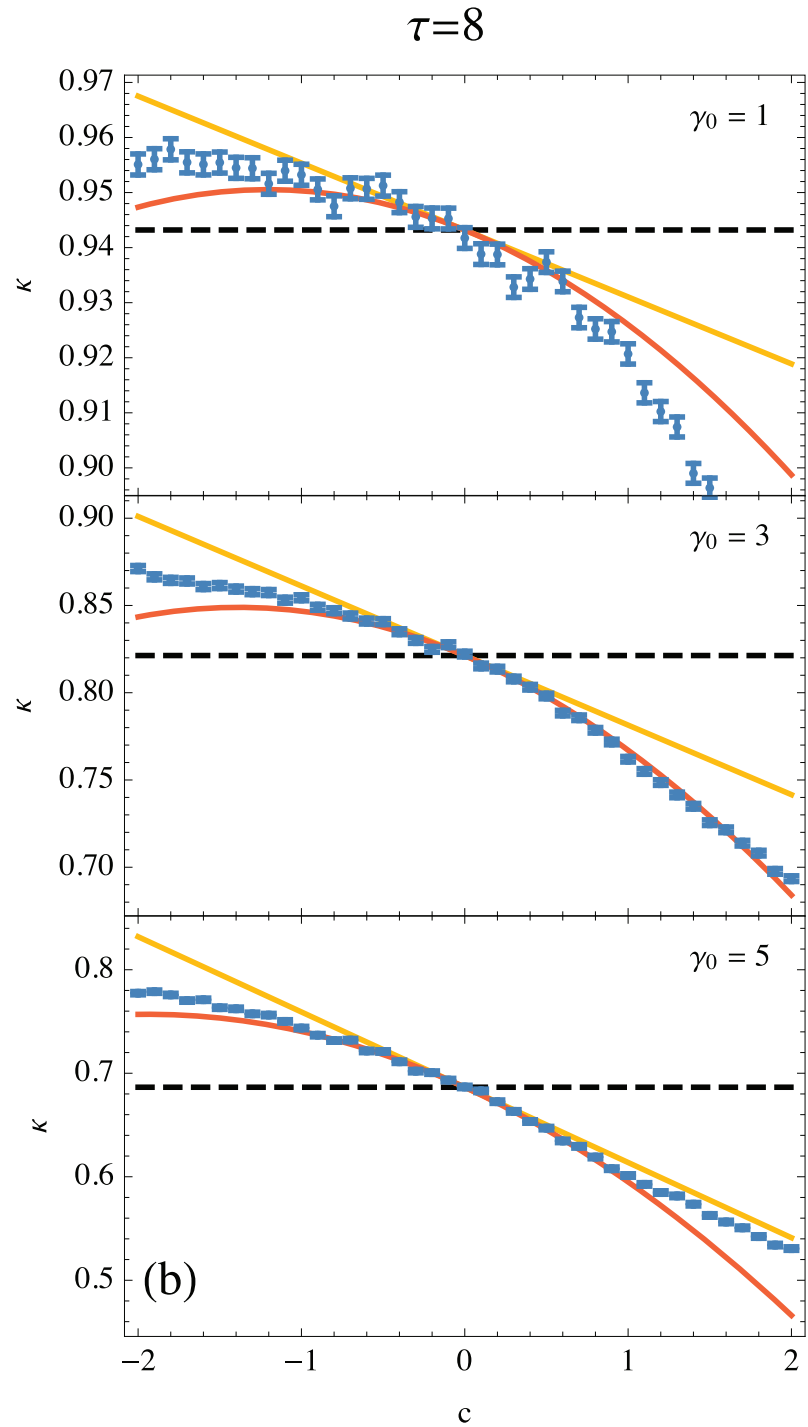

Figure 2. Transmission factor for the two-dimensional model potential (eq. 10) as a function of the coupling strength $c$, for $m=1, k_{\mathrm{B}} T=1, \omega_{1}=1, \omega_{2}=3$. Correlation time (a) $\tau=4$, (b) $\tau=8$ and damping strength $\gamma_{0}=1,3,5$ are as indicated. Numerical simulation results with $1 \sigma$ statistical error bars (blue symbols) are compared to harmonic (Grote-Hynes) approximation (eq. 70) (dashed horizontal line), perturbative results to first-order obtained from eqs (70)+(71) (yellow straight line), and second-order obtained from eqs (70)+(71)+(58c) (red parabola). 
Figure 2 shows the transmission factor for the 2-dof model potential (eq. 10) as a function of the coupling constant $c$ for different values of the memory time $\tau$ and the friction $\gamma_{0}$. As can be seen, the numerical simulations agree with the theoretical results over a wide range of coupling parameters. Even for values of $\kappa$ that differ from the harmonic approximation by about $10 \%$, the agreement with the perturbation theory is still excellent in some cases. As expected in any perturbation theory, the perturbative results eventually deviate from the exact rates as the coupling strength becomes large. In this regime the influence of higher order terms in the asymptotic series (eq. 57) makes itself felt. Though we did not compute higher order corrections in this work, the method can be applied, in principle, to arbitrary order.

\section{Concluding remarks}

In summary, we have presented a method that overcomes the recrossing problem of TST. It identifies reactive trajectories precisely by computing the geometric structures that divide the phase space into reactive and non-reactive parts. More specifically, all the information on the reactivity of the system is encoded in the stable manifold, whose intersection with the DS defines a critical velocity that trajectories must exceed in order to be reactive. This procedure can be used with any choice of DS. The intersection of the stable manifold with a different DS would yield a different critical velocity, but would identify the same trajectories as reactive.

The method reported here has enabled us to obtain analytic corrections to the Grote-Hynes expression for anharmonic multi-dimensional potentials, while providing at the same time a clear geometrical picture of the reaction mechanism.

\section{Acknowledgements}

The research leading to these results has received funding from the Ministerio de Economía y Competitividad under Contract MTM2015-63914-P, ICMAT Severo Ochoa under SEV-2015-0554, and from the European Union's Horizon 2020 research and innovation programme under grant agreement No. 734557.

\section{References}

[1] D G Truhlar, W L Hase and J T Hynes, J. Phys. Chem. 87, 2664 (1983)

[2] D G Truhlar, B C Garrett and S J Klippenstein, J. Phys. Chem. 100, 12771 (1996)

[3] W H Miller, Faraday Discuss. Chem. Soc. 110, 1 (1998)
[4] E Pollak and P Pechukas, J. Chem. Phys. 69, 1218 (1978)

[5] T Uzer, C Jaffé, J Palacián, P Yanguas and S Wiggins, Nonlinearity 15, 957 (2002)

[6] H Waalkens, A Burbanks and S Wiggins, J. Phys. A 37, L257 (2004)

[7] H Waalkens, A Burbanks and S Wiggins, J. Chem. Phys. 121, 6207 (2004)

[8] R G Mullen, J-E Shea and B Peters, The J. Chem. Phys. 140, 041104 (2014)

[9] B C Garrett and D G Truhlar, Variational transition state theory. In C E Dykstra, G Frenking, K S Kim and G E Scuseria, editors, Theory and Applications of Computational Chemistry: The First Forty Years, chapter 5, pages 67-87 (Elsevier 2005)

[10] T Bartsch, R Hernandez and T Uzer, Phys. Rev. Lett. 95 , 058301 (2005)

[11] T Bartsch, T Uzer and R Hernandez, J. Chem. Phys. 123, 204102 (2005)

[12] T Bartsch, T Uzer, J M Moix and R Hernandez, J. Chem. Phys. 124, 244310 (2006)

[13] T Bartsch, T Uzer, J M Moix and R Hernandez, J. Phys. Chem. B 112, 206 (2008)

[14] R Hernandez, T Uzer and T Bartsch, Chem. Phys. 370, 270 (2010)

[15] F Revuelta, T Bartsch, P L Garcia-Muller, R Hernandez, R M Benito and F Borondo, Phys. Rev. E 93, 062304 (2016)

[16] T Bartsch, F Revuelta, R M Benito and F Borondo, Finite-barrier corrections for multidimensional barriers in colored noise (2017) In press

[17] G T Craven, T Bartsch and R Hernandez, Phys. Rev. E 89, 040801(1) (2014)

[18] S Kawai and T Komatsuzaki, J. Chem. Phys. 131, 224505 (2009)

[19] S Kawai and T Komatsuzaki, J. Chem. Phys. 131, 224506 (2009)

[20] F Revuelta, T Bartsch, R M Benito and F Borondo, $J$. Chem. Phys. 136, 091102 (2012)

[21] T Bartsch, F Revuelta, R M Benito and F Borondo, $J$. Chem. Phys. 136, 224510 (2012)

[22] R F Grote and J T Hynes, J. Chem. Phys. 73, 2715 (1980)

[23] P Hänggi, P Talkner and M Borkovec, Rev. Mod. Phys. 62, 251 (1990)

[24] P Pechukas, Statistical approximations in collision theory. In W H Miller, editor, Dynamics of Molecular Collisions, Part B, pages 269-322 (Plenum, New York 1976)

[25] D Chandler, J. Chem. Phys. 68, 2959 (1978)

[26] V I Mel'nikov and S V Meshkov, J. Chem. Phys. 85, 1018 (1986)

[27] E Pollak, H Grabert and P Hänggi, J. Chem. Phys. 91, 4073 (1989)

[28] E Hershkovitz, J. Chem. Phys. 108, 9253 (1998)

[29] E Hershkovitz and R Hernandez, J. Phys. Chem. A 105, 2687 (2001) 
[30] P L García-Müller, F Borondo, R Hernandez and R M Benito, Phys. Rev. Lett. 101, 178302 (2008)

[31] M Ferrario and P Grigolini, J. Math. Phys. 20, 2567 (1979)
[32] P Grigolini, J. Stati. Phys. 27, 283 (1982)

[33] F Marchesoni and P Grigolini, J. Chem. Phys. 78, 6287 (1983)

[34] C C Martens, J. Chem. Phys. 116, 2516 (2002)

[35] T Bartsch, J. Chem. Phys. 131, 124121 (2009) 